\title{
Analysis of cardiac involvement in patients recovered from Covid-19 without troponin elevation, evaluated by cardiovascular magnetic resonance.
}

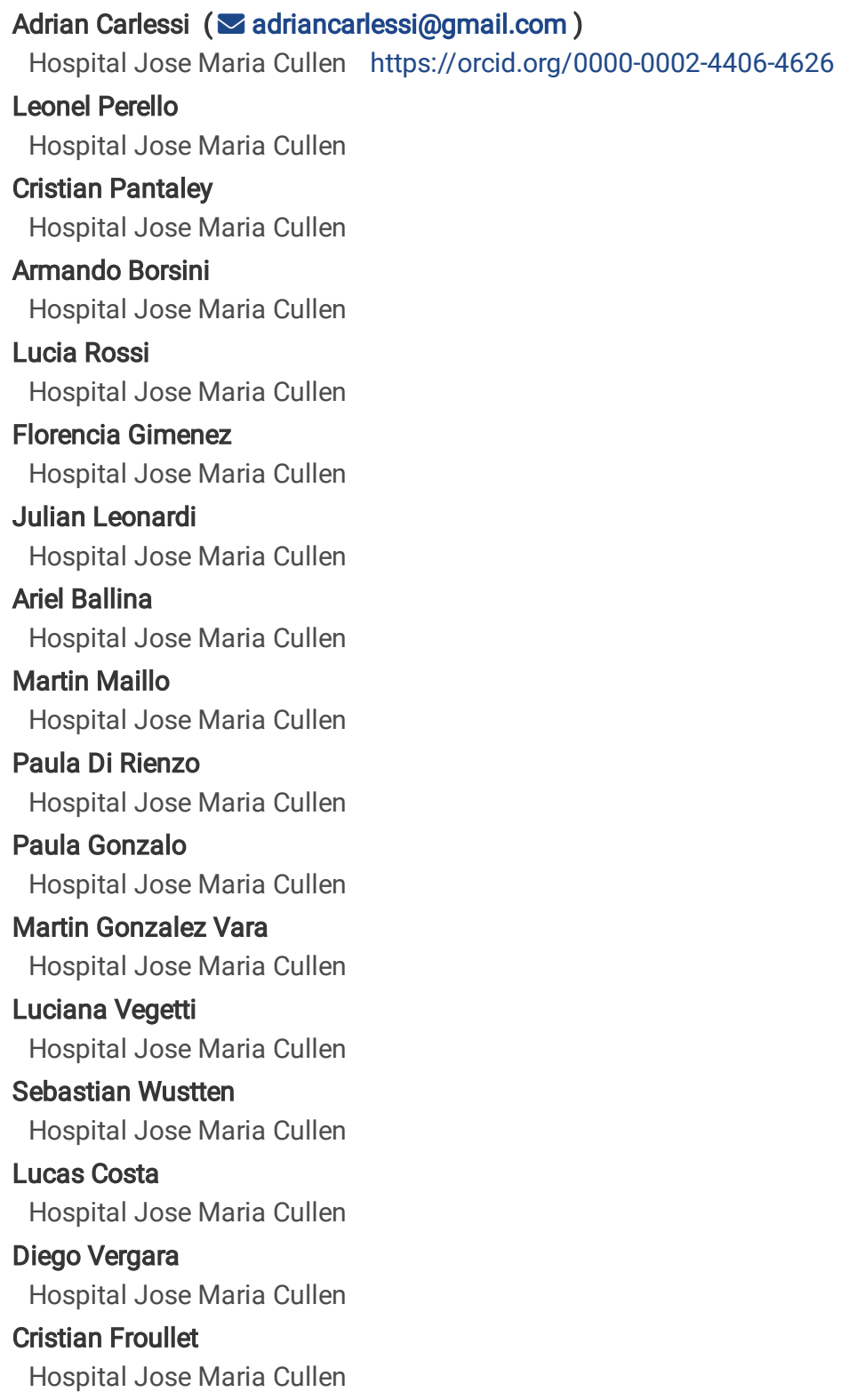




\section{Abstract \\ Background}

The disease caused by coronavirus (COVID-19) affects the cardiovascular system, whether by direct viral aggression or indirectly through systemic inflammation and multiple organ compromise. A widely used method to determine cardiac injury is troponin measurement. The aim of this study is to evaluate the prevalence of cardiac involvement (CINV) in a population recovered from COVID-19, referred to cardiac MRI (CMR), who did not present troponin elevation.

\section{Methods}

There were 156 patients that recovered from COVID-19 and who did not present troponin elevation referred to CMR. CINV was considered to be the presence of: late gadolinium enhancement (LGE), edema, myocarditis, pericarditis, left ventricular systolic dysfunction (LVSD) and/or depressed right ventricular systolic dysfunction (RVSD).

\section{Results}

Prevalence of CINV was $28.8 \%$, being more frequent in men $(p=0.002)$, in patients who required hospitalization $(p=0.04)$ and in those who experienced non-mild cases of infection ( $p=0.007)$. RVSD $(17.9 \%)$ and LVSD $(13.4 \%)$ were the most frequent findings. The rate of myocarditis was $0.6 \%$. LGE manifested in $7.1 \%$ of patients and its presence was related to less left ventricular ejection fraction (LVEF) ( $p=0.0001)$ and right ventricular ejection fraction (RVEF) $(p=0.04)$.

\section{Conclusion}

In patients who recovered from COVID-19, 28.8\% of CINV was found. It was more frequent in men, in patients who required admission and in patients with cases of non-mild infection. The patients that presented LGE had less LVEF and RVSF.

\section{Introduction}

Coronavirus disease 2019 (COVID-19), caused by the coronavirus of the severe acute respiratory syndrome (SARS-CoV-2), emerged in Wuhan, China, in December 2019 in patients who presented with pneumonia by unknown origin[1]. Since then and to this date, when this paper is being written, there have been 134,719,328 cases of COVID-19 developed in the world, with at least 2,915,972 deaths[2]. Although COVID-19 mainly presents with respiratory system compromise, due to the interaction between COVID-19 and the cardiovascular system[3-4], cardiac injury is frequently observed in these patients. The mechanism of cardiac injury is multifactorial, included oxygen supply-demand imbalance without coronary obstruction, myocardial stress, inflammation, microvascular dysfunction, pre-existing atherosclerotic plaque rupture or toxicity by direct viral injury[5]. Studies show a high prevalence of cardiovascular alterations in hospitalized patients[6-7]. Cardiac injury determination through high-sensitivity cardiac troponin shows a worse evolution in patients with increase in this biomarker[8-9].

However, impact on the heart in the mid-term published in different studies is controversial. In the initial reports, a high prevalence of cardiac disease was shown in cardiovascular magnetic resonance (CMR), with elevation in native T1 and T2 mapping being the most frequent finding. These studies included both patients with and without troponin elevation. Late gadolinium enhancement (LGE) was around 31-32\%[10-11]. On the other hand, it was recently observed in patients that were admitted and with elevated troponins at hospital discharge, that LGE prevalence was $33 \%$ of non-ischemic LGE, which may be possibly attributed to COVID-19[12].

The aim of our study is to determine the prevalence of LGE abnormalities in a large sample of patients recovered from COVID-19, who did not present troponin elevation at the time of the study.

\section{Methods}

This is an observational, prospective, single-center study, performed at the Hospital JM Cullen of the city of Santa Fe, Argentina. Consecutive patients from October 2020 to April 2021 who were initially referred for an LGE test and who met the following inclusion criteria were included: 1) signed informed consent 2) confirmed to have infection by SARS-CoV-2 by reverse transcription polymerase chain reaction (RT-PCR); 3 ) more than four weeks after epidemiological discharge; 4) not presenting troponin elevation at the time of the study. The exclusion criteria were: 1) refusal to sign the informed consent; 2) chronic kidney disease with creatinine clearance $\leq 30 \mathrm{ml} / \mathrm{min}$; 3) device or prosthesis not compatible with CMR; 4) claustrophobia. The protocol was analyzed and approved by the institutional bioethical review board. The study protocol conforms to the ethical guidelines of the 1975 Declaration of Helsinki as reflected in a priori approval by the institution's human research committee.

Page 2/9 
According to the severity of COVID-19 symptoms reported by the patient and considering the maximum level of healthcare required, the acute infection was categorized into: mild, moderate, severe and critical, according with the Ordinal Scale for Clinical Improvement (OSCI) proposed by the World Health Organization[13].

\section{LGE protocol and post-process}

LGE was conducted following local institutional guidelines for infections control, with resonator (SIGNA, Creator, GE Healthcare, US). The protocol included:

- Standard long-axis cine imaging (four-, two- and three-chambers, left ventricular outflow tract) and short-axis base-to-tip sweep in expiratory apnea.

- Dark blood imaging on T2-weighted STIR (short-tau inversion recovery) in long axis (four-, two- and three-chambers) and three short-axis views (basal, medial and apical).

- Dark blood imaging on T1-weighted, pre- and post-contrast in the same views as in the T2-weighted sequences.

- After 7-10 minutes of $0.1 \mathrm{mmol} / \mathrm{kg}$ injection of gadobutrol (Gadovist ${ }^{\circledR}$ ), the LGE sequence was made with PSIR (phase-sensitive inversion recovery).

The analysis was made by Cardiac YX (GE Healthcare) for the evaluation of LV volumes and mass; while trabeculae and papillary muscles were excluded. The presence of LGE, edema, myocarditis, pericarditis, left ventricular systolic dysfunction (LVSD) and/or right ventricular systolic dysfunction (RVSD) was classified as cardiac involvement (CINV). Active myocarditis was defined as the presence of non-ischemic LGE (subepicardial or mesocardial location) associated to edema criterion in T2-STIR sequences and/or hyperemia in early post-contrast T1 sequences. The criterion of myocardial edema was established through the myocardial edema index (El), that was defined as the relationship between myocardial signal intensity (SI) and skeletal muscle SI[14]. An EI >2.0 was considered abnormal. Pericarditis criterion was the presence of pericardial LGE or pericardial thickening ( $\geq 4 \mathrm{~mm}$ ) associated to pericardial effusion. LVSD was determined by left ventricular ejection fraction (LVEF) when it was less than $57 \%$ and RVSD when right ventricular ejection fraction (RVEF) was less than $51 \%$ in women and $52 \%$ in men[14]. The location and pattern of LGE injuries in LGE images were evaluated by 2 observers, who reviewed all PSIR images independently.

\section{Statistical analysis}

Statistical analyses were made with IBM SPSS v23. Normality of quantitative variables were evaluated by the Shapiro-Wilk test. To describe quantitative variables, mean or median were used as central tendency measures and standard deviation (SD) or interquartile range (IQR) as corresponding dispersion measures. Qualitative variables were presented as absolute frequencies and their respective percentages. Independent samples t-tests were used to evaluate differences in means between two groups. In the case of not meeting normality criteria, the Mann-Whitney $U$ test was used to compare distributions. Fisher's exact tests and Pearson's chi-squared tests were used to compare differences in rates between qualitative variables. Correlations between quantitative variables were evaluated by means of Pearson correlation coefficient. Confidence intervals of $95 \%(95 \% \mathrm{Cl})$ were attached to the parameters when deemed necessary. a statistical significance was established as 0.05 .

\section{Results}

Clinical characteristics.

The baseline characteristics of patients are shown in Table 1. There were 156 patients included, from whom 55.8\% were men. Age average was 48.4 (SD 13.3). There was $58.9 \%$ of patients with a mild evolution in the acute phase of the disease; only $5.9 \%$ of patients were severe/critical. Patients that required admission were $35.2 \%$. Median and IQR of hospital stay was 10 (5-15) days; while for the ICU it was 6 (4-20). Twenty-one percent of patients reported presenting atypical precordial pain, 18\% palpitations and 515 shortness of breath when undergoing CMR. All these symptoms were not incapacitating and no case required admission. 
Table 1

Clinical characteristics and CMR findings. COPD: Chronic Obstructive

Pulmonary Disease; DBT: Diabetes; RVEF: Right Ventricular Ejection Fraction;

LVEF: Left Ventricular Ejection Fraction; LVMI: Left Ventricular Mass Index;

RVEDVI: Right Ventricular End Diastolic Volume Index; LVEDVI: Left Ventricular

End Diastolic Volume Index; RVESVI: Right Ventricular End Systolic Volume

Index; LVESVI: Left Ventricular End Systolic Volume Index; RVSVI: Right

Ventricular Systolic Volume Index; LVSVI: Left Ventricular Systolic Volume Index.

\begin{tabular}{|c|c|}
\hline \multicolumn{2}{|l|}{ Variable } \\
\hline - Clinical characteristics & Value \\
\hline Age mean $\pm S D$ & $48.4 \pm 13.3$ \\
\hline Male gender $\mathrm{n}(\%)$ & $87(55.8)$ \\
\hline Body mass index mean \pm SD & $28.4 \pm 5.3$ \\
\hline HTN n (\%) & $54(34.6)$ \\
\hline DBT n (\%) & $22(14.1)$ \\
\hline Smoker n (\%) & $48(30.8)$ \\
\hline COPD n (\%) & $5(3.2)$ \\
\hline Previous CAD n (\%) & $5(3.2)$ \\
\hline Previous stroke n (\%) & $2(1.3)$ \\
\hline Charlson comorbidity score media \pm SD & $0.88 \pm 1.37$ \\
\hline Admission due to COVID-19 n (\%) & $55(35.3)$ \\
\hline \multicolumn{2}{|l|}{ COVID-19 severity } \\
\hline Mild n (\%) & $92(59)$ \\
\hline Moderate $\mathrm{n}(\%)$ & $55(35.2)$ \\
\hline Severe/critical n (\%) & $9(5.8)$ \\
\hline Systolic blood pressure in $\mathrm{mmHg}$ mean $\pm \mathrm{SD}$ & $123.8 \pm 16.2$ \\
\hline Diastolic blood pressure in $\mathrm{mmHg}$ mean $\pm \mathrm{SD}$ & $74.9 \pm 9.3$ \\
\hline Heart rate in bpm mean \pm SD & $73.1 \pm 14.4$ \\
\hline \multicolumn{2}{|l|}{ - CMR findings } \\
\hline Days since epidemiological discharge to CMR median (IQR) & $58.5(44-82)$ \\
\hline LVEDVI in $\mathrm{ml} / \mathrm{m}^{2}$ mean $\pm \mathrm{SD}$ & $69.1 \pm 15.6)$ \\
\hline LVESVI in $\mathrm{ml} / \mathrm{m}^{2}$ mean $\pm \mathrm{SD}$ & $26.2 \pm 9.3$ \\
\hline LVSVI in $\mathrm{ml} / \mathrm{m}^{2}$ mean $\pm \mathrm{SD}$ & $42.8 \pm 8.6$ \\
\hline LVEF (\%) mean \pm SD & $62.2 \pm 6.5$ \\
\hline $\mathrm{LVMI}$ in $\mathrm{g} / \mathrm{m}^{2}$ mean $\pm \mathrm{SD}$ & $48.3 \pm 10.6$ \\
\hline $\mathrm{RVEDVI}$ in $\mathrm{ml} / \mathrm{m}^{2}$ mean $\pm \mathrm{SD}$ & $71.5 \pm 16.4$ \\
\hline RVESVI in $\mathrm{ml} / \mathrm{m}^{2}$ mean $\pm \mathrm{SD}$ & $30.5 \pm 9.4$ \\
\hline $\mathrm{RVSVI}$ in $\mathrm{ml} / \mathrm{m}^{2}$ mean $\pm \mathrm{SD}$ & $40.1 \pm 9.8$ \\
\hline RVEF in $\mathrm{ml} / \mathrm{m}^{2}$ mean $\pm \mathrm{SD}$ & $57.5 \pm 6.7$ \\
\hline
\end{tabular}

CMR finding.

Mean and IQR of time from the end of symptoms until performing CMR was 58.5 (44-82) days. In $28.8 \%$ of patients, CINV was observed. Figure 1 shows the prevalence of the different alterations constituting CINV. LVSD and RVSD, with $13.5 \%$ and $17.9 \%$ respectively, were the most frequent 
alterations. Myocardial LGE finding was observed in 11 patients $(7.1 \%)$ and was the most frequent variable found of myocardial tissue involvement. Table 2 shows the characteristics of patients according to the CMR findings. A statistically significant difference was found for gender, with CINV being more prevalent in men, $39.1 \%$ vs $15.9 \%(p=0.002)$. Patients hospitalized due to COVID-19 presented a higher CINV prevalence than those that stayed at home through the disease, $38.2 \%$ vs $23.7 \%(p=0.04)$. In turn, patients that developed non-mild COVID- 19 cases had more CINV than those with mild cases, $40.7 \%$ vs $20.6 \%(p=0.007)$. No difference was observed in the median of days from epidemiological discharge to performance of CMR between the groups, 54 (44-82) days vs $62(43-84)$ days $(p=0.41)$.

Table 2

Clinical parameters of patients that presented CINV and those that had normal CMR

\begin{tabular}{|c|c|c|c|}
\hline Parameter & $\begin{array}{l}\text { NORMAL } \\
111(72.2 \%)\end{array}$ & $\begin{array}{l}\text { CCOMP } \\
45(28.8 \%)\end{array}$ & $P$ value \\
\hline Age mean \pm SD & $47.5 \pm 13.1$ & $50.7 \pm 13.7$ & 0.18 \\
\hline \multicolumn{4}{|l|}{ Gender n (\%) } \\
\hline Male & $53(60.9)$ & $34(39.1)$ & \multirow[t]{2}{*}{0.002} \\
\hline Female & $58(84.1)$ & $11(15.9)$ & \\
\hline Body mass index mean $\pm S D$ & $28.4 \pm 5.5$ & $28.1 \pm 4.7$ & 0.71 \\
\hline \multicolumn{4}{|l|}{ HTN n (\%) } \\
\hline NO & $75(73.5)$ & $27(26.5)$ & \multirow[t]{2}{*}{0.37} \\
\hline YES & $36(66.7)$ & $18(33.3)$ & \\
\hline \multicolumn{4}{|l|}{ DBT n (\%) } \\
\hline NO & $97(72.4)$ & $37(27.6)$ & \multirow[t]{2}{*}{0.40} \\
\hline YES & $14(63.6)$ & $8(36.4)$ & \\
\hline \multicolumn{4}{|l|}{ Smoker n (\%) } \\
\hline NO & $77(71.3)$ & $31(28.7)$ & \multirow[t]{2}{*}{0.95} \\
\hline YES & $34(70.8)$ & $14(29.2)$ & \\
\hline \multicolumn{4}{|l|}{ COPD n (\%) } \\
\hline NO & $108(71.5)$ & $43(28.5)$ & \multirow[t]{2}{*}{0.45} \\
\hline YES & $3(60)$ & $2(40)$ & \\
\hline \multicolumn{4}{|l|}{ Previous CAD n (\%) } \\
\hline NO & $108(71.5)$ & $43(28.5)$ & \multirow[t]{2}{*}{0.45} \\
\hline YES & $3(60)$ & $2(40)$ & \\
\hline \multicolumn{4}{|l|}{ Previous stroke n (\%) } \\
\hline NO & $111(72.1)$ & $43(27.9)$ & \multirow[t]{2}{*}{0.08} \\
\hline YES & $0(0)$ & $2(100)$ & \\
\hline Charlson comorbidity score mean \pm SD & $0.73 \pm 1.2$ & $1.2 \pm 1.6$ & 0.10 \\
\hline \multicolumn{4}{|l|}{ Admission due to COVID-19 n (\%) } \\
\hline NO & $77(76.2)$ & $24(23.8)$ & \multirow[t]{2}{*}{0.04} \\
\hline YES & $34(61.8)$ & $21(38.2)$ & \\
\hline \multicolumn{4}{|l|}{ Non-mild cases } \\
\hline NO & $73(79.3)$ & $19(20.7)$ & \multirow[t]{2}{*}{0.007} \\
\hline YES & $38(59.4)$ & $26(40.6)$ & \\
\hline Days since discharge to CMR median (IQR) & $62(43-84)$ & $54(44-82)$ & 0.64 \\
\hline
\end{tabular}


The location of LGE was subepicardial in $45.4 \%$ of cases, in $36.4 \%$ it was mesocardial, and subendocardial in $18.2 \%$. Patients who presented LGE had a mean LVEF of $54.8 \%$ (SD 8.7\%) and those who did not have LGE had $62.8 \%$ ( $p=0.0001)$, RVEF mean in patients with no LGE was $57.8 \%$ (SD 6.6) and in those with LGE, 53.5\% ( $p=0.04)$ (Fig. 2).

\section{Discussion}

In a cohort of 156 patients recovered from COVID-19, in whom CMR was performed and who did not present troponin elevation at the time of performing the study, $28.8 \%$ of CINV was found. RVSD and LVSD were the most frequently observed alterations, and in most of these, impairment was mild. In our study, the only preexisting condition that showed a significant difference in the presence of CINV was male gender, coinciding with data about a poor evolution in male patients in acute infection [16-17]. The patients who presented non-mild cases and patients who required hospitalization presented more prevalence of CINV, these data differ from the study by Puntmann et al, showing a higher prevalence of CINV (78\%) and this finding is not related to previous comorbidities or the severity of acute clinical symptoms or hospitalization requirement due to COVID-19[11].

The most frequent alterations were those in relation to functionality, and to a lesser extent, those that may reflect direct impact (inflammation, fibrosis, infection) on the heart. These alterations of functionality (with no tissue alterations) may be related to indirect CINV[18]; i.e. dependent on multiple organ dysfunction, with myocardial oxygen supply-demand imbalance caused by the infection, and that are generally observed in nonmild cases, a more frequent compromise than direct viral cardiac injury[19-21].

In our cohort, the prevalence of myocarditis was $0.65 \%$, using the original Lake Louis diagnostic criteria[22]. These were updated in year 2018, with the introduction of T1 and T2 mapping[23]. Only T2 mapping is better than the original Lake Louis criteria in terms of diagnostic sensitivity[24], and when both criteria are compared, the update only improves sensitivity with no impact on specificity. A myocarditis prevalence of $0 \%$ was observed in a series of 16 autopsies and $4.5 \%$ in 201 cases, with the latter being selected cases, which may entail an ever lower prevalence of myocarditis in the general population[25].

In our study, the presence of LGE, a prognostic marker[26], had a prevalence of $7.1 \%$, which is substantially less than that observed in a recent study, where it was $30 \%$. This also included patients with troponin elevation and their values were more elevated in the patients that presented LGE[27]. Unlike this last study, in ours the patients presenting LGE had less LVEF and RVEF ( $p=<0.001$ and $p=0.04$, respectively) than patients that do not present them.

\section{Limitations}

This study presents the limitation of not having CMR data before the COVID-19 infection. Data are shown on a single CMR test of up to 3 months after the infection, and an extended follow-up is essential to determine the progression or regression of the cardiac compromise and its prognostic impact. We do not have T1 and T2 mapping parameters and extracellular volume estimation, which are part of the Lake Louis diagnostic criteria review. However, we did use diagnostic criteria for myocarditis that present a similar specificity.

\section{Conclusion}

Almost a third of patients recovered from COVI-19 with no troponin elevation, cardiac alterations are found. These are more prevalent in patients of the male gender and who have required admission or that presented non-mild cases. Functional alterations (RVSD-LVSD) are most frequent. Myocarditis prevalence was very low. LGE is the most frequent tissue alteration and determinant for LVEF and RVEF.

\section{Declarations}

\section{Acknowledgements}

To Maria Rosa Lombardo,Veronica Monzon and Liset Bringa for their valuable work. To the JM Cullen Hospital and to everyone who has worked to treat this disease and lost their lives.

\section{References}

1. Huang C, Wang Y, Li X et al (2020) Clinical features of patients infected with 2019 novel coronavirus in Wuhan, China. Lancet 395:497-506

2. Dong E, Du H, Gardner L (2020) An interactive web-based dashboard to track COVID-19 in real time. Lancet Infect Dis 20:533-534

3. Knowlton KU (2020) Pathogenesis of SARS-CoV-2 induced cardiac injury from the perspective of the virus. J Mol Cell Cardiol 147:12-17. doi:10.1016/j.yjmcc.2020.08.002 
4. Cheng P, Zhu H, Witteles RM, Wu JC, Quertermous T, Wu SM, Rhee JW. Cardiovascular Risks in Patients with COVID-19: Potential Mechanisms and Areas of Uncertainty. Curr Cardiol Rep. 2020 Apr 29;22(5):34. doi: 10.1007/s11886-020-01293-2. PMID: 32350632; PMCID: PMC7189178

5. Atri D, Siddiqi HK, Lang JP, Nauffal V, Morrow DA, Bohula EA. COVID-19 for the Cardiologist: Basic Virology, Epidemiology, Cardiac Manifestations, and Potential Therapeutic Strategies. JACC Basic Transl Sci. 2020 Apr 10;5(5):518-536. doi: 10.1016/j.jacbts.2020.04.002. PMID: 32292848; PMCID: PMC7151394

6. Wang D, Hu B, Hu C et al (2020) Clinical characteristics of 138 hospitalized patients with 2019 novel coronavirus-infected pneumonia in Wuhan, China. JAMA 323:1061-1069

7. Linghua Fu M, Li X., Yuhao Su M, Jianyong M, Kui Hong et al. Prevalence and impact of cardiac injury on COVID-19: A systematic review and meta-analysis. Clin Cardiol. 2020; 1-8

8. Wei JF, Huang FY, Xiong TY, Liu Q, Chen H, Wang H, Huang $\mathrm{H}$ et al (2020) Acute myocardial injury is common in patients with covid-19 and impairs their prognosis. Heart 106:1154-1159

9. Giustino G, Croft LB, Stefanini GG, Bragato R, Silbiger JJ, Vicenzi M et al (2020) Characterization of Myocardial Injury in Patients With COVID19. J Am Coll Cardiol. Nov 3;76(18):2043-2055. doi: 10.1016/j.jacc.2020.08.069. PMID: 33121710; PMCID: PMC7588179

10. Huang L, Zhao P, Tang D et al. Cardiac involvement in recovered COVID-19 patients identified by magnetic resonance imaging. JACC Cardiovasc Imaging Published online May 12, 2020.doi:10.1016/j.jcmg.2020.05.004

11. Puntmann VO, Carerj ML, Wieters I et al (2020) Outcomes of Cardiovascular Magnetic Resonance Imaging in Patients Recently Recovered From Coronavirus Disease 2019 (COVID-19). JAMA Cardiol 5(11):1265-1273. doi:10.1001/jamacardio.2020.3557

12. Kotecha T, Knight D, Razvi Y, Kumar K, Vimalesvaran K, Thornton G et al (2021) Patterns of myocardial injury in recovered troponin-positive COVID-19 patients assessed by cardiovascular magnetic resonance. Eur Heart J 42:1866-1878. https://doi.org/10.1093/eurheartj/ehab075

13. WHO R\&D Blueprint Novel Coronavirus COVID-19 Therapeutic Trial Synopsis, Accessed, https://www.who.int/blueprint/prioritydiseases/keyaction/COVID19_Treatment_Trial_Design_Master_Protocol_synopsis_Final_18022020.pdf. (Accessed 28 September 2020)

14. Friedrich MG, Sechtem U, Schulz-Menger J et al (2009) Cardiovascular Magnetic Resonance in Myocarditis: A JACC White Paper. J Am Coll Cardiol 53:1475-1487

15. Petersen S, Khanji M, Plein S, Lancellotti P, Bucciarelli-Ducci C (2019) European Association of Cardiovascular Imaging expert consensus paper: a comprehensive review of cardiovascular magnetic resonance normal values of cardiac chamber size and aortic root in adults and recommendations for grading severity. European Heart Journal - Cardiovascular Imaging 20:1321-1331

16. Jian M, Peng B, Wei H, Fei W, Xiao F, De-Min H et al (2020) Gender Differences in Patients With COVID-19: Focus on Severity and Mortality. Frontiers in Public Health Public Health 8:152. doi:10.3389/fpubh.2020.00152

17. Peckham H, de Gruijter NM, Raine $\mathrm{C}$ et al (2020) Male sex identified by global COVID-19 meta-analysis as a risk factor for death and ITU admission. Nat Commun 11:6317. https://doi.org/10.1038/s41467-020-19741-6

18. Giustino G, Pinney SP, Lala A, Reddy VY, Johnston-Cox HA, Mechanick Jl, Halperin JL, Fuster V (2020) Coronavirus and Cardiovascular Disease, Myocardial Injury, and Arrhythmia: JACC Focus Seminar. J Am Coll Cardiol. Oct 27;76(17):2011-2023. doi: 10.1016/j.jacc.2020.08.059. PMID: 33092737; PMCID: PMC7572122

19. Metkus TS, Sokoll LJ, Barth AS et al (2021) Myocardial Injury in Severe COVID-19 Compared With Non-COVID-19 Acute Respiratory Distress Syndrome. Circulation 143(6):553-565. doi:10.1161/CIRCULATIONAHA.120.050543

20. Halushka M, Vander Heide R (2021) Myocarditis is rare in COVID-19 autopsies: cardiovascular findings across 277 postmortem examinations. Cardiovascular Pathology 50:107300. https://doi.org/10.1016/j.carpath.2020.107300

21. Basso C, Leone O, Rizzo S, De Gaspari M, van der Wal C, Aubry A (2020) MC, et al. Pathological features of COVID-19-associated myocardial injury: a multicentre cardiovascular pathology study. Eur Heart J 41:3827-3835

22. Friedrich MG, Sechtem U, Schulz-Menger J, Holmvang G, Alakija P, Cooper LT, White JA, Abdel-Aty H, Gutberlet M, Prasad S, Aletras A, Laissy JP, Paterson I, Filipchuk NG, Kumar A, Pauschinger M, Liu P (2009) International Consensus Group on Cardiovascular Magnetic Resonance in Myocarditis. Cardiovascular magnetic resonance in myocarditis: A JACC White Paper. J Am Coll Cardiol 53:1475-1487. doi:10.1016/j.jacc.2009.02.007

23. Ferreira VM, Schulz-Menger J, Holmvang G et al (2018) Cardiovascular magnetic resonance in nonischemic myocardial inflammation: expert recommendations. J Am Coll Cardiol 72(24):3158-3176

24. Pan JA, Lee YJ, Salerno M. Diagnostic Performance of Extracellular Volume, Native T1, and T2 Mapping Versus Lake Louise Criteria by Cardiac Magnetic Resonance for Detection of Acute Myocarditis: A Meta-Analysis. Circ Cardiovasc Imaging. 2018 Jul;11(7):e007598. doi: 10.1161/CIRCIMAGING.118.007598. PMID: 30012826; PMCID: PMC6192699

25. Kawakami R, Sakamoto A, Kawai K et al (2021) Pathological Evidence for SARS-CoV-2 as a Cause of Myocarditis: JACC Review Topic of the Week. J Am Coll Cardiol 77(3):314-325. doi:10.1016/j.jacc.2020.11.031 
26. Aquaro GD, Perfetti M, Camastra G, Monti L, Dellegrottaglie S, Moro et al (2017) Cardiac Magnetic Resonance Working Group of the Italian Society of Cardiology. Cardiac MR With Late Gadolinium Enhancement in Acute Myocarditis With Preserved Systolic Function: ITAMY Study. J Am Coll Cardiol. Oct 17;70(16):1977-1987. doi: 10.1016/j.jacc.2017.08.044. PMID: 29025554

27. Wang H, Li R, Zhou Z et al (2021) Cardiac involvement in COVID-19 patients: mid-term follow up by cardiovascular magnetic resonance. J Cardiovasc Magn Reson 23:14. https://doi.org/10.1186/s12968-021-00710-x

\section{Figures}

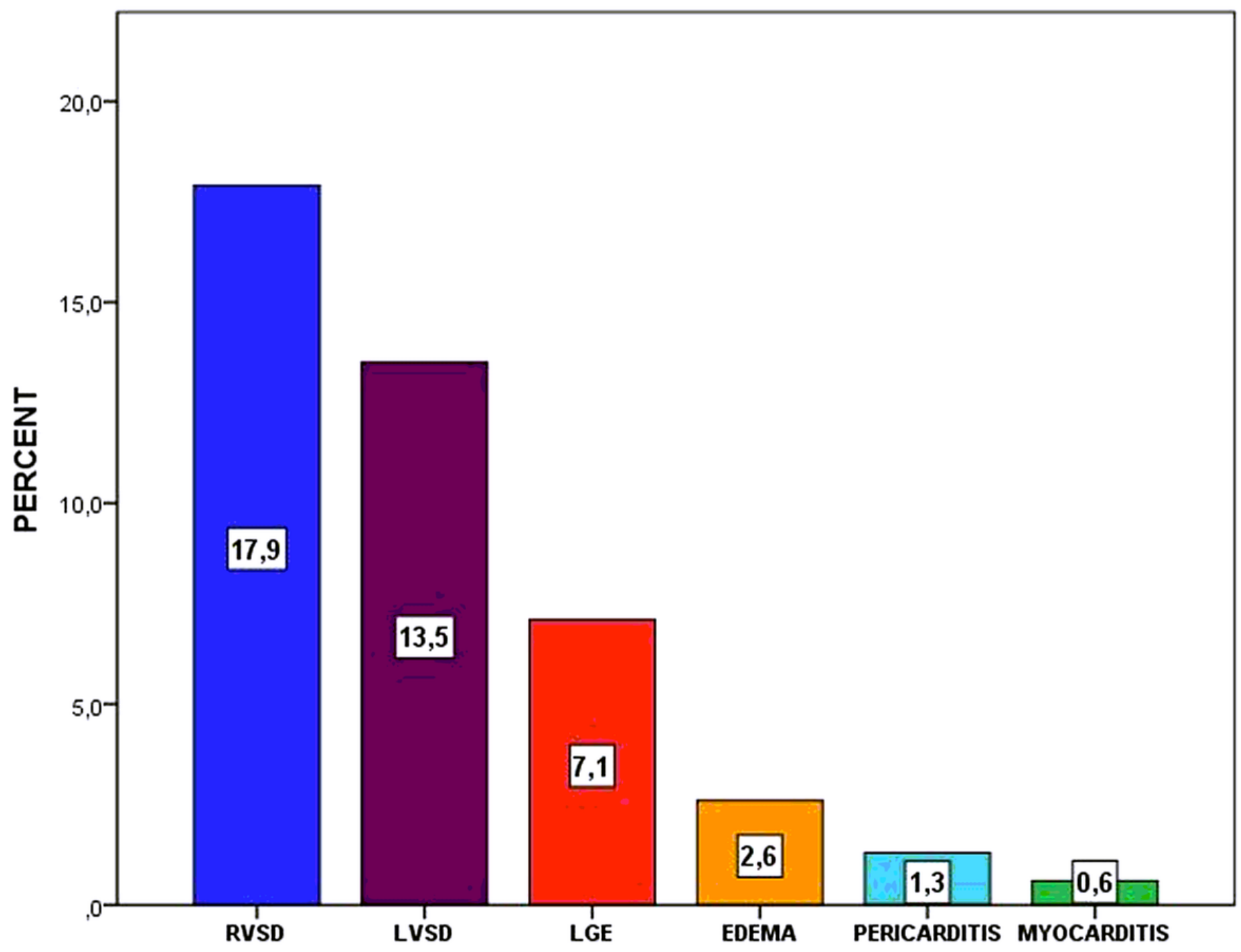

Figure 1

Findings in CMR. Components of CINV 

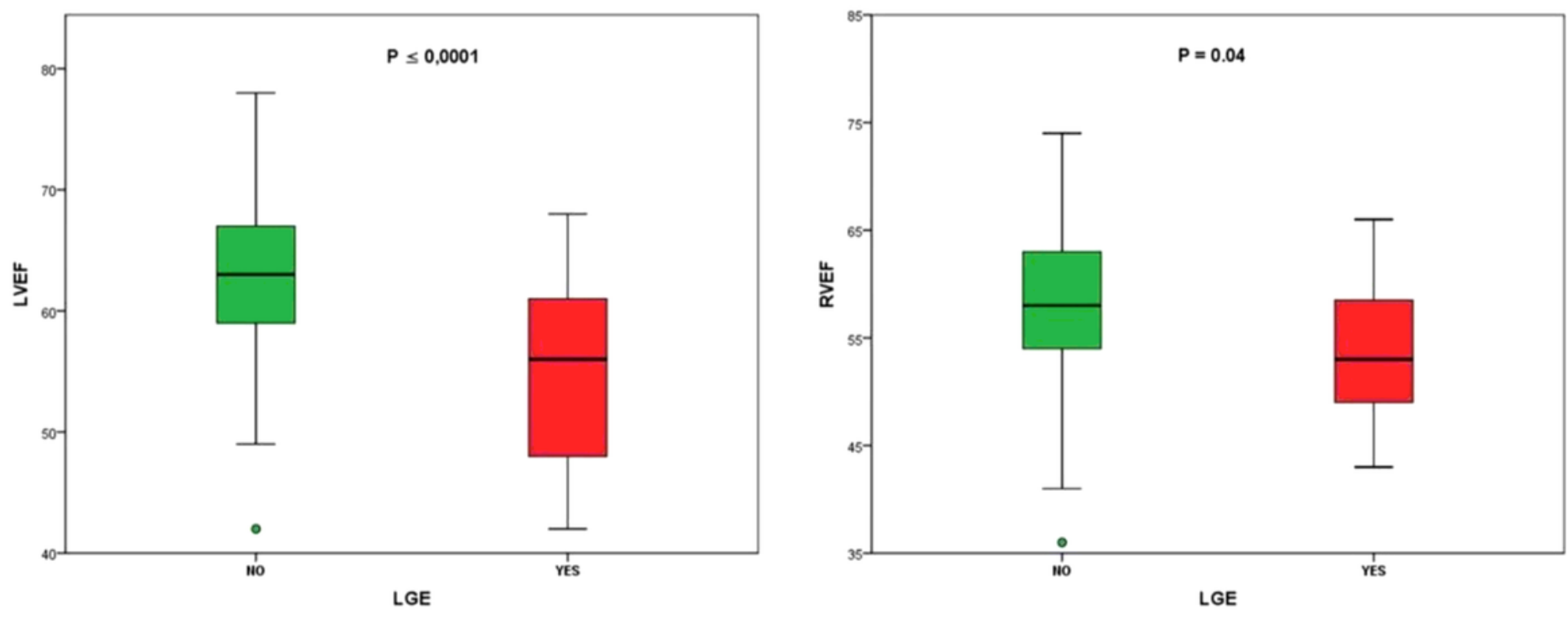

Figure 2

LVEF and RVEF according to the presence of LGE. 